\title{
SOME TAUBERIAN PROPERTIES OF HÖLDER TRANSFORMATIONS
}

\section{AMNON JAKIMOVSKI ${ }^{1}$}

1. Introduction. The result which follows was proved by me, recently, in [1], ${ }^{2}$ Theorem (9.2). If, for some $\alpha>-1$, the sequence $\left\{s_{n}\right\}, n=0,1,2, \cdots$, is summable $A^{(\alpha)}$ to $s$, that is

$$
\sum_{n=0}^{\infty}\left(\begin{array}{c}
n+\alpha \\
n
\end{array}\right) s_{n} z^{n}
$$

is convergent in the unit circle and

$$
\lim _{x \uparrow 1}(1-x)^{\alpha+1} \sum_{n=0}^{\infty}\left(\begin{array}{c}
n+\alpha \\
n
\end{array}\right) s_{n} x^{n}=s \quad(|s|<+\infty),
$$

and, for some pair $\beta, \gamma$ of real numbers with $\beta<\gamma$,

$$
\lim _{n \rightarrow \infty}\left(h_{n}^{(\beta)}-h_{n}^{(\gamma)}\right)=0
$$

then

$$
\lim _{n \rightarrow \infty} h_{n}^{(\beta)}=s,
$$

when generally $\left\{h_{n}^{(\delta)}\right\}$ denotes the sequence of the Hölder transform of order $\delta$ of $\left\{s_{n}\right\}$.

The Hölder transform of order $\alpha\left\{h_{n}^{(\alpha)}\right\}$ (or, in short, the $(H, \alpha)$ transform), where $\alpha$ is a real number, is defined as the Hausdorff transform generated by the sequence $\mu_{n}=(n+1)^{-\alpha}, n=0,1,2, \cdots$. It is known that the Hölder transformations are regular for $\alpha \geqq 0$. We say, too, that a sequence $\left\{s_{n}\right\}$ is summable Hölder to $s$ if it is summable $(H, \alpha)$ to $s$ for some real number $\alpha$.

In this note we obtain an extension of my above result to the case

Received by the editors January 19, 1955 and, in revised form, June 23, 1955.

1 This note is in part a part of a manuscript On relations between methods of summability of infinite series and integrals submitted as a thesis for the degree of Ph.D. to the Hebrew University, Jerusalem.

2 Numbers in brackets refer to the bibliography at the end of this note.

- Concepts and propositions mentioned or used in this paper without definition or proof are to be found in Hardy's book Divergent series, Oxford University Press. 
where $\left\{h_{n}^{(\gamma)}\right\}$ is replaced by certain linear combinations of Hölder transforms as well as some other results of similar type (see $\$ \S 8$ and 9).

In the proof of the extensions just mentioned we use some properties of general and special Hausdorff transformations as well as two tauberian propositions for $A^{(\alpha)}$ methods of summability, formulated below in $\$ \S 2-7$.

2. Some properties of the $J^{(\alpha, \beta)}$ and $J\left(\alpha ; \begin{array}{l}\beta, \gamma \\ a, b\end{array}\right)$ transformations.

For any pair $\alpha, \beta$ of real numbers, with $\alpha<\beta$, we call (see $[1$, p. 378]) the linear transform $\left\{t_{n}^{(\alpha, \beta)}\right\}$ of $\left\{s_{n}\right\}$,

$$
t_{0}^{(\alpha, \beta)}=h_{0}^{(\alpha)} ; \quad t_{n}^{(\alpha, \beta)}=h_{0}^{(\alpha)}+\sum_{v=1}^{n} \frac{h_{v}^{(\alpha)}-h_{v}^{(\beta)}}{(\beta-\alpha) v}, \quad n>0,
$$

the $J^{(\alpha, \beta)}$ transform of $\left\{s_{n}\right\} .\left\{s_{n}\right\}$ is called summable $J^{(\alpha, \beta)}$ to $s$ if $\lim _{n \rightarrow \infty} t_{n}^{(\alpha, \beta)}=s$.

The following two properties of $J^{(\alpha, \beta)}$ transforms were proved by me in [1, Theorem (9.1) and Theorem (9.2)].

Theorem A. For any pair $\alpha, \beta$ of real numbers, with $\alpha<\beta$, the $J^{(\alpha, \beta)}$ transform of $\left\{s_{n}\right\}$ is a Hausdorff transform generated by $\left\{\mu_{n}\right\}$, where $\mu_{0}=1 ; \mu_{n}=\left((n+1)^{-\alpha}-(n+1)^{-\beta}\right) /(\beta-\alpha) n, n>0$.

Theorem B. For any pair $\alpha, \beta$ of real numbers, with $\alpha<\beta$, the $J^{(\alpha, \beta)}$ method of summability is equivalent to the $(H, \alpha+1)$ method; thus, for $-1 \leqq \alpha<\beta$, the $J^{(\alpha, \beta)}$ transformation is regular.

A simple consequence of Theorem A and Theorem B is

THEOREM 2.1. Let $a, b, \alpha, \beta$ and $\gamma$ be five real numbers satisfying $a+b=1, \alpha<\beta<\gamma, 0 \leqq a$ and $a \alpha+b \beta>0$. Let $\left\{s_{n}\right\}$ be an arbitrary sequence. The linear transform

$$
\left\{U_{n}^{(\alpha ; \underset{a}{\beta}, \boldsymbol{\gamma})}\right\}
$$

defined by

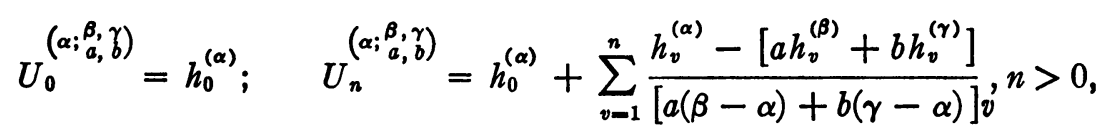

is a Hausdorff transform of $\left\{s_{n}\right\}$ generated by the sequence $\left\{\mu_{n}\right\}, \mu_{0}=1$; $\mu_{n}=\{[a(\beta-\alpha)+b(\gamma-\alpha)] n\}^{-1} \cdot\left[(n+1)^{-\alpha}-a(n+1)^{-\beta}-b(n+1)^{-\gamma}\right]$, $n>0$. 
Definition. The linear Hausdorff transform

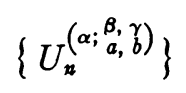

of the sequence $\left\{s_{n}\right\}$ defined in Theorem 2.1 is called the

$$
J\left(\alpha ; \begin{array}{l}
\beta, \gamma \\
a, b
\end{array}\right)
$$

transform of $\left\{s_{n}\right\}$.

The transformation

$$
J\left(\alpha ; \begin{array}{c}
\beta, \gamma \\
a, b
\end{array}\right)
$$

is, clearly, regular whenever $\alpha \geqq-1$.

3. Some properties of Mellin transforms. Given a function $f(t)$ of bounded variation for $0 \leqq t \leqq 1$, then the function $F(z)$ defined by $F(z)=\int_{0}^{1} t^{z} d f(t)$ is called the Mellin transform of the function $f(t)$. A result concerning Mellin transforms, which we use later, is

Theorem C (PitT's THeorem). Let the function T(z) be a Mellin transform of the function $\alpha(t)$ where $\alpha(t)$ is of bounded variation in $\langle 0,1\rangle$ satisfying $\alpha(0)=\alpha(+0)=0 ; \alpha(1)=1$. If $|T(z)| \geqq d>0$ for $\operatorname{Re} z \geqq 0$ then $\{T(z)\}^{-1}$ is also a Mellin transform of some function $\beta(t)$ of bounded variation in $\langle 0,1\rangle$ satisfying $\beta(0)=\beta(+0)=0 ; \beta(1)=1$.

For a proof of Theorem $\mathrm{C}$ and a generalization of it see $\left[3, \mathrm{pp} .178^{-}\right.$ 179].

The main result of this section is

THEOREM 3.1. Let $a, b, \alpha$ and $\beta$ be real numbers satisfying $a+b=1$, $0<\alpha<\beta, 0 \leqq a$ and $a \alpha+b \beta>0$. Then the sequence $\left\{\mu_{n}\right\}$, defined by $\mu_{0}$ $=1 ; \mu_{n}=\alpha \cdot[a \alpha+b \beta]^{-1} \cdot\left[1-(n+1)^{-\alpha}\right] \cdot\left[1-a(n+1)^{-\alpha}-b(n+1)^{-\beta}\right]^{-1}$, $n>0$, is a regular moment sequence.

In the proof of Theorem 3.1 we use two auxiliary propositions, Lemma 3.1 and Lemma 3.2; the first proposition

LEMMA 3.1. Let $a, b, \alpha$ and $\beta$ be four real numbers satisfying $a+b=1$, $0<\alpha<\beta, 0 \leqq a$ and $a \alpha+b \beta>0$. Then the function $T(z)$ defined by $T(z)=(a \alpha+b \beta) \cdot \alpha^{-1} \cdot\left[1-(z+1)^{-\alpha}\right] \cdot\left[1-a(z+1)^{-\alpha}-b(z+1)^{-\beta}\right]$, is $a$ Mellin transform of a function $\gamma(t)$ of bounded variation on $\langle 0,1\rangle$ satisfying $\gamma(0)=\gamma(+0)=0 ; \gamma(1)=1$.

follows simply from 
Lemma A. For $0<\alpha, \beta$ the function $T(z)$ defined by

$$
T(z)=\frac{\beta}{\alpha} \cdot \frac{1-(z+1)^{-\alpha}}{1-(z+1)^{-\beta}}
$$

$\left((z+1)^{-\alpha}=e^{-\alpha \cdot \log (z+1)}\right.$, where we choose for $\log (z+1)$ its principal branch) is a Mellin transform of a function $\alpha(t)$, of bounded variation in $\langle 0,1\rangle$, satisfying $\alpha(0)=\alpha(+0)=0 ; \alpha(1)=1$.

For a proof of Lemma A see [1, Lemma 9.1].

It is easy to prove the second auxiliary proposition

Lemma 3.2. With the suppositions of Lemma 3.1 on $a, b, \alpha$, and $\beta$ and for the function $T(z)$ defined there, there exists a positive number $g$ such that for $\operatorname{Re} z \geqq 0,|T(z)| \geqq g>0$.

The proof of Theorem 3.1 follows immediately by combining Lemma 3.2 with Theorem C.

4. Further properties of $J\left(\alpha ; \begin{array}{l}\beta, \gamma \\ a, b\end{array}\right)$ methods of summability.

In this section we prove a property of the

$$
J\left(\alpha ; \begin{array}{l}
\beta, \gamma \\
a, b
\end{array}\right)
$$

method of summability which is formulated in

Theorem 4.1. For all real numbers $a, b, \alpha, \beta$ and $\gamma$ satisfying $a+b=1, \alpha<\beta<\gamma, 0 \leqq a$ and $a \alpha+b \beta>0$ the

$$
J\left(\alpha ; \begin{array}{l}
\beta, \gamma \\
a, b
\end{array}\right)
$$

method of summability is equivalent to the $(H, \alpha+1)$ method of summability.

Proof. Since both the transformations

$$
J\left(\alpha ; \begin{array}{l}
\beta, \gamma \\
a, b
\end{array}\right)
$$

and $J^{(\alpha, \beta)}$ are Hausdorff transformations and, by Theorem B, the $J^{(\alpha, \beta)}$ and $(H, \alpha+1)$ methods of summability are equivalent, it is enough (by a well known theorem of Hausdorff) to show that the following two sequences $\left\{\mu_{n}\right\}$ and $\left\{\lambda_{n}\right\}$, where 


$$
\begin{array}{r}
\mu_{0}=1 ; \mu_{n}=\frac{\beta-\alpha}{a(\beta-\alpha)+b(\gamma-\alpha)} \cdot \frac{1-(n+1)^{-(\beta-\alpha)}}{1-a(n+1)^{-(\beta-\alpha)}-b(n+1)^{-(\gamma-\alpha)}} \\
n>0, \\
\lambda_{0}=1 ; \lambda_{n}=\frac{a(\beta-\alpha)+b(\gamma-\alpha)}{\beta-\alpha} \cdot \frac{1-a(n+1)^{-(\beta-\alpha)}-b(n+1)^{-(\gamma-\alpha)}}{1-(n+1)^{-(\beta-\alpha)} \quad}, \\
n>0,
\end{array}
$$

are regular moment sequences; which is precisely the content of Theorem 3.1 and Lemma 3.1, respectively. Q.E.D.

5. On products of summability methods. The following result on the product of $A^{(\alpha)}$ and regular Hausdorff methods of summability was proved by me in [1] (the special case $\alpha=0$ of this proposition is due to O. Szász. See [4]).

THEOREM D. If, for some $\alpha>-1,\left\{s_{n}\right\}$ is summable $A^{(\alpha)}$ to $s$ and $\left\{t_{n}\right\}$ is any regular Hausdorff transform of $\left\{s_{n}\right\}$ then $\left\{t_{n}\right\}$ is also summable $A^{(\alpha)}$ to $s$.

6. Two tauberian properties of the $A^{(\alpha)}$ methods of summability. The two tauberian theorems for $A^{(\alpha)}$ methods of summability, formulated below, are known (compare [2, Theorem 3.1 and its proof]).

THEOREM E. If, for some $\alpha>-1,\left\{s_{n}\right\}$ is summable $A^{(\alpha)}$ to $s$ and $s_{n}-s_{n-1}=O(1 / n), n \rightarrow \infty$, then $\left\{s_{n}\right\}$ is summable $(H,-1+\epsilon)$ to $s$ for each $\epsilon>0$.

Theorem F. If, for some $\alpha>-1,\left\{s_{n}\right\}$ is summable $A^{(\alpha)}$ to $s$ and $s_{n}-s_{n-1}=O_{L}(1 / n), n \rightarrow \infty$, then $\left\{s_{n}\right\}$ is convergent to $s$.

7. A tauberian theorem for Hölder summability. The tauberian theorem, for Hölder summability, stated below is also interesting in itself.

THEOREM 7.1. Let $\left\{s_{n}\right\}$ be summable Hölder to $s$. Then, given a real number $\alpha$ arbitrarily a necessary and sufficient condition for $\left\{s_{n}\right\}$ to be summable $(H, \alpha)$ is that for a system of $2 n$ real numbers $a_{1}, a_{2}, \cdots, a_{n}$, $\alpha_{1}, \alpha_{2}, \cdots, \alpha_{n}$ satisfying $\alpha<\alpha_{1}<\cdots<\alpha_{n}$ and $a_{1}+a_{2}+\cdots+a_{n}=1$ holds

$$
\lim _{m \rightarrow \infty}\left\{h_{m}^{(\alpha)}-\left[a_{1} h_{m}^{\left(\alpha_{1}\right)}+a_{2} h_{m}^{\left(\alpha_{2}\right)}+\cdots+a_{n} h_{m}^{\left(\alpha_{n}\right)}\right]\right\}=0 .
$$

An argument similar to that used in the proof of Theorem 2.1 of my paper [1] establishes Theorem 7.1 as well as a similar result for Cesàro summability. I do not state the last result explicitly here. 
8. Tauberian theorems for the $A^{(\alpha)}$ methods of summability. We can prove now the generalizations just mentioned in $\$ 1$.

THEOREM 8.1. Let $a, b, \alpha, \beta$ and $\gamma$ be five real numbers satisfying $a+b=1, \alpha<\beta<\gamma, 0 \leqq a$ and $a(\beta-\alpha)+b(\gamma-\alpha)>0$. Then necessary and sufficient conditions for the $(H, \alpha)$ summability of $\left\{s_{n}\right\}$ are

$$
\lim _{n \rightarrow \infty}\left\{h_{n}^{(\alpha)}-\left[a h_{n}^{(\beta)}+b h_{n}^{(\gamma)}\right]\right\}=0
$$

and that for some $\delta>-1\left\{s_{n}\right\}$ is summable $A^{(\delta)}$.

Proof. It is easy to see that (8.1) and the $A^{(\delta)}$ summability are necessary for the summability considered. Now we show that (8.1) together with the $A^{(\delta)}$ summability are sufficient for the same purpose. First we suppose that $\alpha \geqq 0$. By Theorem $D$ and Theorem 2.1 we have for

$$
\left\{U_{*}^{(\alpha ; \beta, \gamma, \delta)}\right\}
$$

the

$$
J\left(\alpha ; \begin{array}{c}
\beta, \gamma \\
a, b
\end{array}\right)
$$

transform of $\left\{s_{n}\right\}$,

$$
\lim _{x \uparrow 1}(1-x)^{\delta+1} \sum_{n=0}^{\infty}\left(\begin{array}{c}
n+\delta \\
n
\end{array}\right) \cdot U_{n}^{\left(\begin{array}{c}
\alpha ; \\
a, j
\end{array}\right)} x^{n}=s .
$$

From Theorem 2.1 and Theorem $\mathrm{E}$ we infer, by (8.1), that $\left\{s_{n}\right\}$ is summable

$$
J\left(\alpha ; \begin{array}{l}
\beta, \gamma \\
a, b
\end{array}\right)
$$

to $s$. Hence, by Theorem $4.1,\left\{s_{n}\right\}$ is summable $(H, \alpha+1)$ to $s$; thus Theorems 7.1 and 8.1 yield $\lim _{n \rightarrow \infty} h_{n}^{(\alpha)}=s$. Our theorem is proved in the case $\alpha \geqq 0$. If, now, $\alpha<0$ then there exists a positive integer $d$ such that $\alpha+d>0$, hence, by taking the $(H, d)$ transform of the sequence $\left\{h_{n}^{(\alpha)}-\left[a h_{n}^{(\beta)}+b h_{n}^{(\gamma)}\right]\right\}$, possessing the limit zero, we obtain $h_{n}^{(\alpha+d)}-\left[a h_{n}^{(\beta+d)}+b h_{n}^{(\gamma+d)}\right] \rightarrow 0, n \rightarrow \infty$, and the last relation shows, by the first part of the proof, that $\left\{s_{n}\right\}$ is summable Hölder; whence the rest of the proof follows now by Theorem 7.1. Q.E.D.

Theorem 8.1 and the other results of this section have already been proved in [1] in the case $a=1$. 
An argument similar to that used in the proof of Theorem 8.1 (but exploiting now Theorem $\mathrm{E}$ entirely and also using Theorem $\mathrm{F}$ as in [1, proof of Theorem 6.2 and proof of Theorem 6.3]) yields

THEOREM 8.2. Let $a, b, \alpha, \beta$ and $\gamma$ be five real numbers satisfying $a+b=1, \alpha<\beta<\gamma, 0 \leqq a$ and $a(\beta-\alpha)+b(\gamma-\alpha)>0$. If, for some real number $\delta>-1,\left\{s_{n}\right\}$ is summable $A^{(\delta)}$ and (i) $h_{n}^{(\alpha)}-\left[a h_{n}^{(\beta)}+b h_{n}^{(\gamma)}\right]$ $=O_{L}(1)$, as $n \rightarrow \infty$, then $\left\{s_{n}\right\}$ is summable $(H, \alpha+1)$ to $s$. If, further, (ii) $h_{n}^{(\alpha)}-\left[a h_{n}^{(\beta)}+b h_{n}^{(\gamma)}\right]=O(1)$, as $n \rightarrow \infty$, then $\left\{s_{n}\right\}$ is summable $(H,-1+\alpha+\epsilon)$ to $s$ for each $\epsilon>0$.

The argument used in the proof of Theorem 5.2 of [1] together with Theorem 3.1 of the present paper enable us to obtain

THEOREM 8.3. Let the nine real numbers $a, a^{\prime}, b, b^{\prime}, \alpha, \beta, \beta^{\prime}, \gamma$ and $\gamma^{\prime}$ satisfy $\alpha<\beta<\gamma, \alpha<\beta^{\prime}<\gamma^{\prime}, a+b=a^{\prime}+b^{\prime}=1, a(\beta-\alpha)+b(\gamma-\alpha)>0$ and $a^{\prime}\left(\beta^{\prime}-\alpha\right)+b^{\prime}\left(\gamma^{\prime}-\alpha\right)>0$. If

$$
\lim _{n \rightarrow \infty} \frac{h_{n}^{(\alpha)}-\left[a h_{n}^{(\beta)}+b h_{n}^{(\gamma)}\right]}{a(\beta-\alpha)+b(\gamma-\alpha)}=l
$$

then also

$$
\lim _{n \rightarrow \infty} \frac{h_{n}^{(\alpha)}-\left[a^{\prime} h_{n}^{\left(\beta^{\prime}\right)}+b^{\prime} h_{n}^{\left(\gamma^{\prime}\right)}\right]}{a^{\prime}\left(\beta^{\prime}-\alpha\right)+b^{\prime}\left(\gamma^{\prime}-\alpha\right)}=l .
$$

REMARK. Combining arguments of $[1, \S 7]$, with propositions of this note we may obtain results for the Borel method of summability, similar to those of this section, which generalize those of $[1, \S 7]$. I do not state these results explicitly here.

9. Further properties of the Hölder transformations. Given $2 n+1$ real numbers $a_{1}, a_{2}, \cdots, a_{n}, \alpha, \alpha_{1}, \alpha_{2}, \cdots, \alpha_{n}$ satisfying $\alpha<\alpha_{1}$ $<\alpha_{2}<\cdots<\alpha_{n}$ and $a_{1}+a_{2}+\cdots+\alpha_{n}=1$ we call the sequence

defined by

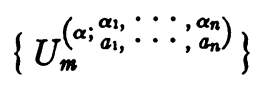

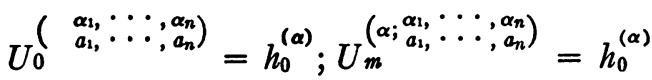

$$
\begin{aligned}
& +\sum_{v=1}^{m} \frac{h_{v}^{(\alpha)}-\left[a_{1} h_{v}^{\left(\alpha_{1}\right)}+\cdots+a_{n} h_{v}^{\left(\alpha_{n}\right)}\right]}{\left[a_{1}\left(\alpha_{1}-\alpha\right)+\cdots+a_{n}\left(\alpha_{n}-\alpha\right)\right] v}, n>0,
\end{aligned}
$$

the 


$$
J\left(\alpha ; \begin{array}{c}
\alpha_{1}, \cdots, \alpha_{n} \\
a_{1}, \cdots, a_{n}
\end{array}\right)
$$

transform of the sequence $\left\{s_{n}\right\}$. Using results of the previous sections it is easy to show that the

$$
J\left(\alpha ; \begin{array}{c}
\alpha_{1}, \cdots, \alpha_{n} \\
a_{1}, \cdots, a_{n}
\end{array}\right)
$$

transformation is a Hausdorff transformation generated by the sequence $\left\{\mu_{m}\right\}$, where

$$
\begin{array}{r}
\mu_{0}=1 ; \quad \mu_{m}=\frac{(m+1)^{-\alpha}-\left[a_{1}(m+1)^{-\alpha_{1}}+\cdots+a_{n}(m+1)^{-\alpha_{n}}\right]}{\left[a_{1}\left(\alpha_{1}-\alpha\right)+\cdots+a_{n}\left(\alpha_{n}-\alpha\right)\right] \cdot m}, \\
m>0 .
\end{array}
$$

Taking this into consideration we can prove now one of the main results of this section, namely

TheORem 9.1. Given $2 n+1$ real numbers $a_{1}, a_{2}, \cdots, a_{n}, \alpha, \alpha_{1}$, $\alpha_{2}, \cdots, \alpha_{n}$ satisfying $\alpha<\alpha_{1}<\alpha_{2}<\cdots<\alpha_{n}, 0<\min \left(a_{1}, \cdots, a_{n}\right)$ and $a_{1}+a_{2}+\cdots+a_{n}=1$, the

$$
J\left(\alpha ; \begin{array}{c}
\alpha_{1}, \cdots, \alpha_{n} \\
a_{1}, \cdots, a_{n}
\end{array}\right)
$$

method of summability is equivalent to the $(H, \alpha+1)$ method of summability; thus the

$$
J\left(\alpha ; \begin{array}{c}
\alpha_{1}, \cdots, \alpha_{n} \\
a_{1}, \cdots, a_{n}
\end{array}\right)
$$

method of summability is regular if $\alpha \geqq-1$.

The proof of Theorem 9.1 is the same as that of Theorem 4.1 but now we have to use a simple modification of Lemma 3.2.

Now we mention a result which is more general than Theorem 9.1. This generalization is obtained by using the following argument.

Let $\alpha, \beta$ and $\gamma$ be three real numbers satisfying $\alpha<\beta<\gamma$. If $a(u)$ is a nondecreasing and bounded function in $\beta \leqq u \leqq \gamma$ which satisfies $a(\gamma)-a(\beta)=1$ then call

$$
\left.\left\{U_{n}^{(\alpha ; \beta, \gamma(u)}\right)\right\}
$$

defined by 


$$
U_{0}^{(\alpha ; \underset{a(u)}{\beta, \gamma})}=h_{0}^{(\alpha)} ; U_{n}^{(\alpha ; \underset{a(u)}{\beta, \gamma})}=h_{0}^{(\alpha)}+\sum_{v=1}^{n} \frac{h_{v}^{(\alpha)}-\int_{\beta}^{\gamma} h_{n}^{(t)} d a(t)}{v \cdot \int_{\beta}^{\gamma}(t-\alpha) d a(t)}, n>0,
$$

the

$$
J\left(\alpha ; \begin{array}{l}
\beta, \gamma \\
a(u)
\end{array}\right)
$$

transform of $\left\{s_{n}\right\}$. It is easy to see that this transformation is a Hausdorff transformation generated by the sequence $\left\{\mu_{n}\right\}$ where

$$
\mu_{0}=1 ; \quad \mu_{n}=\frac{(n+1)^{-\alpha}-\int_{\beta}^{\gamma}(n+1)^{-t} d a(t)}{n \cdot \int_{\beta}^{\gamma}(t-\alpha) d a(t)}, \quad n>0 .
$$

In the same way that Theorem 4.1 was proved we may prove

THEOREM 9.2. If (i) $\alpha, \beta$ and $\gamma$ are three real numbers satisfying $\alpha<\beta<\gamma$ and (ii) $a(u)$ is $a$ nondecreasing and bounded function in $\beta \leqq u \leqq \gamma$ satisfying $a(\gamma)-a(\beta)=1$, then the

$$
J\left(\alpha ; \begin{array}{l}
\beta, \gamma \\
a(u)
\end{array}\right)
$$

method of summability is equivalent to the $(H, \alpha+1)$ method; thus the

$$
J\left(\alpha ; \begin{array}{l}
\beta, \gamma \\
a(u)
\end{array}\right)
$$

method of summability is regular if $\alpha \geqq-1$.

It is easy to see that if in Theorem 8.1, Theorem 8.2 and Theorem 8.3 we replace expressions of the form $h_{m}^{(\alpha)}-\left[a h_{m}^{(\beta)}+b h_{m}^{(\gamma)}\right]$ by expressions of the form $h_{m}^{(\alpha)}-\int_{\beta}^{\gamma} h_{m}^{(u)} d a(u)$, where $a(u)$ is a nondecreasing and bounded function in $\beta \leqq u \leqq \gamma$ satisfying $a(\gamma)-a(\beta)=1$, and expressions of the form $a(\beta-\alpha)+b(\gamma-\alpha)$ by expressions of the form $\int_{\beta}^{\gamma}(u-\alpha) d a(u)$, then the modified conclusions of these modified theorems remain valid.

\section{BIBLIOGRAPHY}

1. A. Amir (Jakimovski), Some relations between the methods of summability of 
Abel, Borel, Cesdro, Hölder and Hausdorff, Journal d'Analyse Mathématique vol. 3 (1953-1954) pp. 346-381.

2. On a converse of Abel's theorem, Proc. Amer. Math. Soc. vol. 3 (1952) pp. 244-256.

3. W. W. Rogosinski, On Hausdorf methods of summability, Proc. Cambridge Philos. Soc. vol. 38 (1942) pp. 166-192.

4. O. Szász, On the product of two summability methods, Annales de la Societé Polonaise de Mathématique vol. 25 (1952-1953) pp. 75-84.

Tel-Aviv, IsRael

\section{ON A THEOREM OF J. L. WALSH}

\section{ARYEH DVORETZKY}

1. In a recent paper [1] J. L. Walsh proved, among other results, the following theorem:

Let the functions $f_{n}(x)(n=1,2, \cdots)$ and $f(x)$ be $p$ times differentiable in the interval $a<x<b$ and let $f_{n}(x)$ converge to $f(x)$ in this interval. Then, given any point $x_{0} \in(a, b)$ there exists a sequence of points $x_{n} \in(a, b)$ such that

$$
\lim _{n=\infty} x_{n}=x_{0}, \quad \lim _{n=\infty} f_{n}^{(p)}\left(x_{n}\right)=f^{(p)}\left(x_{0}\right) .
$$

The main purpose of this short note is to show that "in general" there exists a sequence $x_{n}$ satisfying the first condition of (1) and for which $f_{n}^{(p)}\left(x_{n}\right)=f^{(p)}\left(x_{0}\right)$ for all sufficiently large $n$; and when this does not occur then for the corresponding $n$ not only (1) holds but $f_{n}^{(p)}(x)$ is close, in a sense which will be made precise, to $f^{(p)}\left(x_{0}\right)$ in the neighborhood of $x_{0}$. While doing this we shall replace the convergence assumption by a considerably weaker one.

2. Theorem. Let $f(x)$ and $f_{n}(x)(n=1,2, \cdots)$ be $p$ times differentiable in the interval $a<x<b$ and let

$$
\lim _{n=\infty} \operatorname{Inf}_{x \in I, y \in I}\left|f_{n}(y)-f(x)\right|=0
$$

for every open sub-interval I of $(a, b)$. Then, given any point $x_{0} \in(a, b)$, the sequence $N=\{n\}$ can be written as a union of two (not necessarily both infinite) sequences $N_{1}=\left\{n_{1}\right\}$ and $N_{2}=\left\{n_{2}\right\}$ in such a way that

Received by the editors March 28, 1955 and, in revised form, May 23, 1955. 